\title{
Cannabis in the Treatment of Rheumatic Diseases: Suggestions for a Reasoned Approach
}

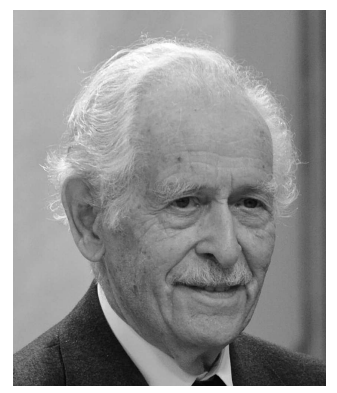

On April 1, 2014, Health Canada announced a new approach to the permitted use of herbal cannabis as a medication ("medical marijuana"). Physicians are now required to write prescriptions, which the patients must have filled by producers licensed by Health Canada who will sell standard preparations of known purity. Numerous medical organizations, such as the Canadian Medical Association, the College of Family Physicians of Canada, and the Canadian Ophthalmological Society, have expressed their disapproval either of the use of cannabis as a medication, or of the requirement for physicians to write prescriptions for a substance that has not yet been formally approved as a safe and efficacious medication by Health Canada itself.

A general assessment of these issues and an appeal for a calm and reasoned approach to the medical uses of cannabis and pure cannabinoids was recently published elsewhere ${ }^{1}$. However, the debate has not ended, and in this issue of The Journal a further statement of opposition can be found to the new regulations on behalf of the Canadian Rheumatology Association $^{2}$. The reasons given for opposing the requirement for physicians to write prescriptions for cannabis are essentially the same as those given in the statements by other medical organizations: that the efficacy and safety of cannabis have not yet been demonstrated in well-designed clinical trials in patients with chronic rheumatic and arthritic conditions; that physicians should not be obliged to write prescriptions for a drug that does not yet have defined recommended dosage levels; that the steady increase in potency of contemporary cannabis puts the patients at potentially increased risk; and that the medical prescription of cannabis under these conditions is a violation of the basic principles of good medical practice.

These are not trivial concerns, and the medical groups that have raised them have had evident reason for doing so. However, in reacting to the new regulations announced by Health Canada, it is useful to bear in mind the reasons for their enactment, and the probable, as opposed to the theoretically possible, degree of risk arising from medically directed and supervised use of cannabis for the treatment of rheumatic and arthritic conditions.

The creation of the Medical Marijuana Access program was forced on Health Canada by a judicial ruling that if there was reason to believe that cannabis might be helpful in the treatment of disease, it would be a violation of the Canadian Charter of Rights and Freedoms ${ }^{3}$ to not permit access to it. The threat was that if Health Canada did not devise a means of allowing access to it within 1 year, the Canadian drug control legislation would be invalid ${ }^{4}$. Under the circumstances, the original access regulations were a stop-gap measure in which Health Canada took responsibility for deciding who would be granted access to cannabis for medical purposes, and physicians were required only to provide information about the patient's diagnosis and to indicate that the patient believed that cannabis might be helpful. While this was clearly a less demanding role for the physician than the new role, one may legitimately ask whether the physician is not a better judge of the patient's "need" for cannabis than an arm's-length committee that has no direct knowledge of the patient. It is a difficult and worrying role for the physician, for the reasons set out by Fitzcharles and Jamal, but medicine has never been an easy profession and physicians are surely better qualified than nonphysicians to practice it.

The burden may not in fact be quite as great as it might appear. There is a very comprehensive review of the literature on therapeutic uses of cannabis that is readily available to physicians on Health Canada's website ${ }^{5}$. Additionally, in response to the expressed concerns of physicians, Health Canada is currently developing a variety of communications to physicians that will provide expert advice about practical matters such as recommended dosage and duration of treatment with cannabis for the different potential indications, emphasizing the need for careful screening of appropriate patients, and pointing out important contraindications to its use.

One important and puzzling omission remains, however.

\section{See Expanding medical marijuana in Canada, page 143}


Fitzcharles and Jamal correctly note the great variability in potency of different cannabis preparations, and express the hope that Health Canada regulations under the new system will "provide a more standardized product for patients medicating with herbal cannabis." ${ }^{2}$ Unfortunately, this does not appear to be the case, since the licensed producers are free to select the strains that they choose to produce, and the regulations do not specify the cannabinoid composition nor the delta-9-tetrahydrocannabinol (THC) content. Physicians can perhaps play an important role in making emphatically clear to the government the importance of having truly standardized preparations.

A factor that may make the prescription of cannabis less worrisome for physicians is that the scientific base underlying its use in rheumatic diseases is not as limited as Fitzcharles and Jamal suggest. Relief of chronic musculoskeletal pain is not mediated only by cannabinoid action on brain cannabinoid type 1 receptors $(\mathrm{CB} 1)^{6,7,8,9}$. An important development has been the recognition and elucidation of the antiinflammatory actions of the endocannabinoid system, and of the role of inflammatory mechanisms in the generation of peripheral pain sensation. Cannabinoids have been shown to act also through CB2, TRPV1, GPR55, and descending fiber 5-HT3 receptors, and to inhibit release of interleukins, tumor necrosis factor- $\alpha$, nitric oxide, and other proinflammatory factors from macrophages and immune cells, which are involved in the generation of pain in animal models of osteoarthritis ${ }^{10,11,12}$. The variety of different mechanisms through which cannabinoids act to relieve pain and inflammation warrants greater confidence that cannabis therapy may have a useful place in rheumatology, even though there is still work to be done in translating from basic studies to clinical use ${ }^{13}$.

In the treatment of rheumatic diseases, just as in the other therapeutic applications of cannabis and cannabinoids, they are not the treatments of first choice. For example, as analgesics, they are less potent and less versatile than opioids. Therefore, physicians are fully justified in trying other treatments first, even for patients who ask for cannabis therapy, and in resorting to the latter only when the preferred treatment does not give satisfactory results. Alternatively, cannabis and cannabinoids have much lower acute toxicity from overdose than opioids or other analgesics, and have not caused any proven deaths attributable to cannabis itself. Therefore, there is the possibility of using cannabis or cannabinoids in combination with other agents rather than as an exclusive form of treatment. It has been shown, for example, that combining smaller doses of cannabinoid and opioid has resulted in a positive analgesic effect in human patients and fewer side effects than a larger dose of either drug alone ${ }^{14}$. This approach may offer greater confidence in the use of small doses of cannabis or cannabinoid, and may offer greater flexibility of treatment options.

It is important to recognize that smoking herbal cannabis is not to be equated with taking pure cannabinoids by mouth. One must therefore question why Canadian rheumatologists "have expressed a lack of confidence in their knowledge and ability to responsibly advise patients on the rational use of cannabinoid agents, including herbal cannabis" ${ }^{2}$. Pure THC (dronabinol) and nabilone did indeed undergo the detailed examination of efficacy and safety that is required of all approved drugs, and have been legally available for prescription use for many years. Given the "orphan drug" status of cannabidiol (CBD) in the United States, it seems likely that it will also become an approved drug in the near future. All these agents are free of the uncertainty of dosage and the adverse effects of smoking on the pulmonary system that are valid concerns in relation to herbal cannabis. The great majority of clinical studies have been done with oral cannabinoids rather than smoked cannabis. The physician therefore has the option of trying such cannabinoids first, and resorting to smoked cannabis only for patients who are already using it nonmedically, or who require the rapid onset of action that smoking provides.

As Fitzcharles and Jamal correctly note, most of the information concerning adverse effects of cannabis has come from studies of nonmedical users, and principally from longterm heavy users ${ }^{15,16}$. It is highly probable that under conscientious medical supervision, the norm will be to use relatively low doses and for limited periods of time. Therefore, the adverse effects are likely to be relatively minor, as has been the case in recent clinical trials ${ }^{17,18,19}$. The main reason for dropout in earlier trials in patients with cancer, most of whom had not experienced cannabis before, was the occurrence of undesired psychoactivity. Since CBD and possibly other cannabinoids have analgesic and antiinflammatory activity, but not the undesired psychoactivity that is exerted through CB1 receptors, they offer further options for improved therapeutic use.

The medical use of cannabis is not without problems, and rheumatologists, like other physicians, must use due caution in prescribing it under the new regulations. There are strong reasons for not prescribing it to children, adolescents, and pregnant women ${ }^{20,21}$, or to patients with previous history of abuse of psychoactive drugs. However, physicians can prescribe it with reasonable expectation of benefit in carefully selected and monitored cases, until the development of newer and much more selective endocannabinoid modulators makes the use of herbal cannabis a thing of the past ${ }^{1}$.

HAROLD KALANT, MD, PHD, FRSC, Department of Pharmacology \& Toxicology, University of Toronto and Centre for Addiction and Mental Health, Toronto, Ontario, Canada

Address correspondence to Dr. H. Kalant, Department of Pharmacology \& Toxicology, Medical Sciences Building, University of Toronto, 1 King's College Circle, Toronto, Ontario, Canada M5S 1A8.

E-mail: harold.kalant@utoronto.ca

Personal non-commercial use only. The Journal of Rheumatology Copyright @ 2015 . All rights reserved. 


\section{REFERENCES}

1. Kalant H. Marihuana: Medicine, addictive substance, or both? A common-sense approach to the place of cannabis in medicine. Can J Addict 2013;4(3):4-8.

2. Fitzcharles M, Jamal S. Expanding medical marijuana access in Canada: considerations for the rheumatologist. J Rheumatol 2015;42:143-5

3. Canadian Charter of Rights and Freedoms. Catalogue No. S2-215/2003. Ottawa: Minister of Public Works and Government Services Canada; 2003.

4. Canada: Pot; A Case For Fair Play. Source: Toronto Star. Publication date: December 12, 1997. [Internet. Accessed June 12, 2014.] Available from: www.cfdp.ca/dec1097.htm

5. Health Canada. Cannabis (marihuana, marijuana) and the cannabinoids. February 2013. [Internet. Accessed June 6, 2014.] Available from: www.hc-sc.gc.ca/dhp-mps/alt_formats/pdf/ marihuana/med/infoprof-eng.pdf

6. Rahn EJ, Hohmann AG. Cannabinoids as pharmacotherapies for neuropathic pain: from the bench to the bedside. Neurotherapeutics 2009;6:713-37.

7. Anand P, Whiteside G, Fowler CJ, Hohmann AG. Targeting CB2 receptors and the endocannabinoid system for the treatment of pain. Brain Res Rev 2009 Apr;60:255-66.

8. Kress M, Kuner R. Mode of action of cannabinoids on nociceptive nerve endings. Exp Brain Res 2009;196:79-88.

9. McDougall JJ. Peripheral analgesia: hitting pain where it hurts. Biochimica et Biophysica Acta 2011;1812:459-67.

10. Zhang RX, Ren K, Dubner R. Osteoarthritis pain mechanisms: basic studies in animal models. Osteoarthritis Cartilage 2013;21:1308-15.

11. Stein C, Machelska H. Modulation of peripheral sensory neurons by the immune system: implications for pain therapy. Pharmacol Rev 2011;63:860-81.
12. Namazi MR. Cannabinoids, loratidine and allopurinol as novel additions to the antipsoriatic ammunition. J Eur Acad Dermatol Venereol 2005;19:319-22.

13. Pacher P, Kunos G. Modulating the endocannabinoid system in human health and disease - successes and failures. FEBS J 2013;280:1918-43.

14. Elikkottil J, Gupta P, Gupta K. The analgesic potential of cannabinoids. J Opioid Manag 2009;5:341-57.

15. Kalant H. Adverse effects of cannabis on health: an update on the literature since 1996. Prog Neuropsychopharmacol Biol Psychiatry 2004;28:849-63.

16. Hall W, Degenhardt L. Adverse health effects of non-medical cannabis use. Lancet 2009;374:1383-91.

17. Ware MA, Wang T, Shapiro S, Robinson A, Ducruet T, Huynh T, et al. Smoked cannabis for chronic neuropathic pain: a randomized clinical trial. CMAJ 2010;182:E694-701.

18. Wang T, Collet JP, Shapiro S, Ware MA. Adverse effects of medical cannabinoids: a systematic review. CMAJ 2008;178:1669-78.

19. Ben Amar M. Cannabinoids in medicine: a review of their therapeutic potential. J Ethnopharmacol 2006;105:1-25.

20. Meier MH, Caspi A, Ambler A, Harrington H, Houts R, Keefe RS, et al. Persistent cannabis users show neuropsychological decline from childhood to midlife. Proc Natl Acad Sci U S A 2012;109:E2657-64.

21. Fried PA. Cannabis use during pregnancy: its effects on offspring from birth to young adulthood. In: Preece PM, Riley E, editors. Alcohol, drugs and medication in pregnancy: the long-term outcome for the child. London, UK: Mac Keith Press, Blackwell Publishing; 2011:153-68.

J Rheumatol 2015;42:146-8; doi:10.3899/jrheum.140683 